\title{
'||||||||||||||||||||||||||||||||||||||||||||||||||||||||||||||||||||||||.
}

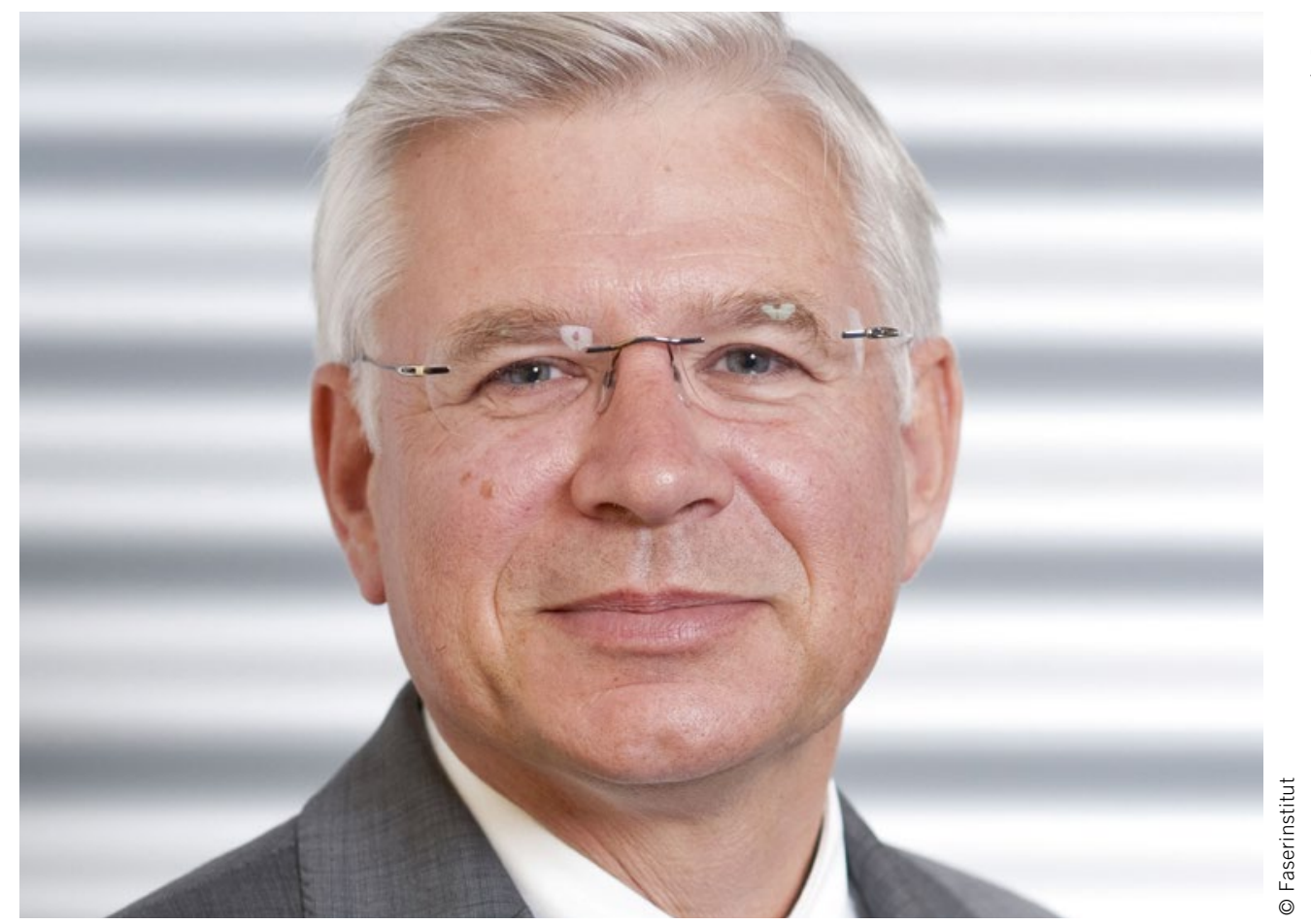

Prof. Dr. Axel Herrmann

Geschäftsführer CTC GmbH Stade und Vorsitzender des VDI-Fachbereichs Luft- und Raumfahrttechnik

\section{Digitale Transformation}

Die globalen Megatrends - wie das Streben nach Individualisierung, Nachhaltigkeit, Digitalisierung und Konnektivität erfordern einen immensen Wandel des Status quo der Luftund Raumfahrtbranche. Connected Aviation ist dabei nur eine Umschreibungen dessen, wie sich schon heute die Relation zwischen Passagier, Betreiber, Instandhalter, Flughafen und Flugzeughersteller verändert und weiterentwickelt. Einige dieser Trends werden von den Akteuren nicht nur positiv bewertet - stellen sie doch regelmäßig etablierte Wertschöpfungsketten infrage oder machen diese gar obsolet.

Gleichzeitig ist aber gerade die Luft- und Raumfahrtbranche einer der größten Profiteure des nahezu unbegrenzten Potenzials der voranschreitenden Digitalisierung, die den Unternehmen zahlreiche Chancen für neue Wertschöpfungsformen und erweiterten Marktzugang bietet. Betrachtet man das Industrial Internet of Things (IIoT) und Industrie 4.0 als eine mögliche vierte industrielle Revolution, gehört nicht nur die hochtechnisierte Raumfahrt, sondern erstmals auch die zivile Luftfahrt zu den Early Adoptern. In nicht wenigen Technologien wie der Virtuellen Realität oder den Additiven Fertigungsverfahren treibt die Branche Innovationen aktiv voran. Als Beispiel sei der Abgleich von gefertigten Bauteilen oder Baugruppen in Echtzeit mit digitalen CAD-Modellen durch Augmented oder Mixed Reality-Applikationen genannt. Noch als Proof of Concept in vielen Branchen in Untersuchung, wird diese Technologie bei Airbus bereits erfolgreich in Serie zur Montageerleichterung, Vollständigkeitskontrolle und Qualitätssicherung eingesetzt. Genauso sind Big Data oder Predictive Maintenance für die Triebwerkshersteller mit einer rund um die Uhr fernüberwachten Motorenflotte keine Modeworte mehr.

Um die Adaption der digitalen Technologien in der Fertigung zu beschleunigen, gilt es sichere und flexible Schnittstellen zur stabilen Anbindung an ERP-, PLM- und MES-Systeme verfügbar zu machen. Ein straffes Regulierungsprogramm und hohe Sicherheitsanforderungen erfordern langzeitorientierte, skalierbare und durchgängige Lösungen. Gerade im Fertigungsumfeld der Luftfahrt ist daher geboten, sich nicht nur an den ansprechenden Verheißungen der täglich potenteren Technologien zu orientieren und dadurch Gefahr zu laufen, ungeeignete Anwendungsgebiete auszuwählen. Eine erfolgreiche, die Wertschöpfung verbessernde Umsetzung bedarf einer engen Abstimmung mit allen involvierten Parteien und ein tiefes Prozessverständnis. Nutzerfreundlichkeit und -akzeptanz, Robustheit und messbare Verbesserung - nur einige Faktoren, die hohe Priorität genießen sollten.

Das Zusammenbringen der verschiedenen Institutionen, Anbieter und Anwender, um die Luft- und Raumfahrtbranche auf ihrem Weg in das digitale Zeitalter zu begleiten - auch dies wird Inhalt und Ziel des Fachbeirats Luft- und Raumfahrttechnik der VDI-Gesellschaft Fahrzeug- und Verkehrstechnik sein. 